University of New Hampshire

University of New Hampshire Scholars' Repository

Space Science Center

Institute for the Study of Earth, Oceans, and

Space (EOS)

2000

\title{
Gamma ray measurements of the 1991 November 15 solar flare
}

\author{
M B. Arndt \\ University of New Hampshire - Main Campus \\ K Bennett \\ ESTEC \\ A Connors \\ University of New Hampshire - Main Campus \\ Mark L. McConnell \\ University of New Hampshire - Main Campus, mark.mcconnell@unh.edu \\ G Rank \\ Max-Planck-Institut für extraterrestrische Physik
}

See next page for additional authors

Follow this and additional works at: https://scholars.unh.edu/ssc

Part of the Astrophysics and Astronomy Commons

\section{Recommended Citation}

Gamma ray measurements of the 1991 November 15 solar flare Arndt, Martina B. and Bennett, Kevin and Connors, Alanna and McConnell, Mark and Rank, Gerhard and Ryan, James M. and Schönfelder, Volker and Suleiman, Raid and Young, C. Alex, AIP Conference Proceedings, 510, 569-573 (2000),

DOl:http://dx.doi.org/10.1063/1.1303267

This Conference Proceeding is brought to you for free and open access by the Institute for the Study of Earth, Oceans, and Space (EOS) at University of New Hampshire Scholars' Repository. It has been accepted for inclusion in Space Science Center by an authorized administrator of University of New Hampshire Scholars' Repository. For more information, please contact Scholarly.Communication@unh.edu. 


\section{Authors}

M B. Arndt, K Bennett, A Connors, Mark L. McConnell, G Rank, James M. Ryan, V Schonfelder, R Suleiman, and $C$ A. Young 


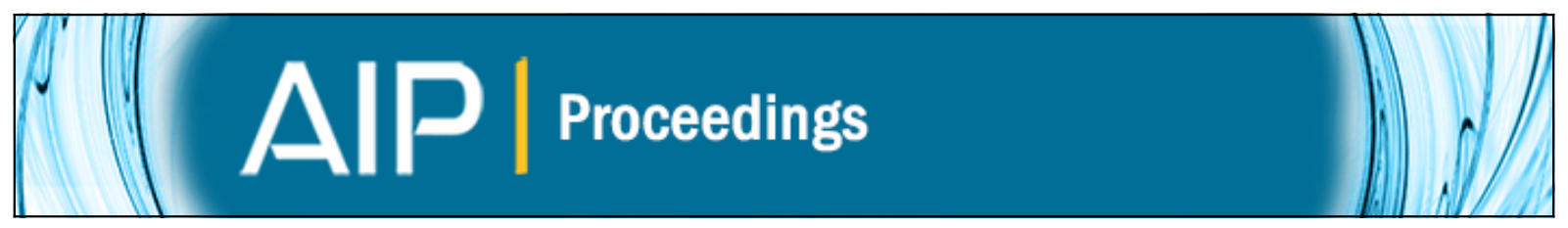

\section{Gamma ray measurements of the 1991 November 15 solar flare}

Martina B. Arndt, Kevin Bennett, Alanna Connors, Mark McConnell, Gerhard Rank, James M. Ryan, Volker

Schönfelder, Raid Suleiman, and C. Alex Young

Citation: AIP Conference Proceedings 510, 569 (2000); doi: 10.1063/1.1303267

View online: http://dx.doi.org/10.1063/1.1303267

View Table of Contents: http://scitation.aip.org/content/aip/proceeding/aipcp/510?ver=pdfcov

Published by the AIP Publishing

\section{Articles you may be interested in}

X- and gamma-ray observations of the 15 November 1991 Solar Flare

AIP Conf. Proc. 587, 618 (2001); 10.1063/1.1419473

High energy processes in solar flares

AIP Conf. Proc. 522, 401 (2000); 10.1063/1.1291742

Energetic proton spectra in the 11 June 1991 solar flare

AIP Conf. Proc. 510, 564 (2000); 10.1063/1.1303266

Accelerated-particle spectral variability in the 1991 June 11 solar flare

AIP Conf. Proc. 510, 559 (2000); 10.1063/1.1303265

The MeV cosmic gamma-ray background measured with SMM

AIP Conf. Proc. 510, 471 (2000); 10.1063/1.1303252 


\title{
Gamma Ray Measurements of the 1991 November 15 Solar Flare
}

\author{
Martina B. Arndt ${ }^{1}$, Kevin Bennett ${ }^{2}$, Alanna Connors ${ }^{1}$, Mark \\ McConnell ${ }^{1}$, Gerhard Rank ${ }^{3}$, James M. Ryan ${ }^{1}$, Volker Schönfelder ${ }^{3}$, \\ Raid Suleiman ${ }^{1}$, and C. Alex Young ${ }^{1}$ \\ ${ }^{1}$ Space Science Center, University of New Hampshire, Durham, NH 03824 \\ ${ }^{2}$ Astrophysics Division of ESA/Estec, NL-2200 AG Noordwijk, The Netherlands \\ ${ }^{3}$ Max-Plank Institute für Extraterrestrische Physik, D-8046, Garching Germany
}

\begin{abstract}
The 1991 November 15 X1.5 flare was a well observed solar event. Comprehensive data from ground-based observatories and spacecraft provide the basis for a contextual interpretation of gamma-ray spectra from the Compton Gamma Ray Observatory (CGRO). In particular, spectral, spatial, and temporal data at several energies are necessary to understand the particle dynamics and the acceleration mechanism(s) within this flare. X-ray images, radio, Ca XIX data and magnetograms provide morphological information on the acceleration region $[4,5]$, while gamma-ray spectral data provide information on the parent ion spectrum. Furthermore, time profiles in hard Xrays and gamma-rays provide valuable information on temporal characteristics of the energetic particles. We report the results of our analysis of the evolution of this flare as a function of energy $(\sim 25 \mathrm{keV}-2.5 \mathrm{MeV})$ and time. These results, together with other high energy data (e.g. from experiments on Yohkoh, Ulysses, and PVO) may assist in identifying and understanding the acceleration mechanism(s) taking place in this event.
\end{abstract}

\section{INTRODUCTION}

The 1991 November 15 X1.5 solar flare was a well observed event. It occurred near disk center at S13W19 $\left(\sim 23^{\circ}\right.$ heliocentric angle $)$ in active region NOAA 6919. $\mathrm{X}$-ray emission commenced $\sim 2235: 00 \mathrm{UT}$, peaked $\sim 2237: 30 \mathrm{UT}$, and lasted on the order of 5 minutes. High energy observations of this event between $\sim 25 \mathrm{keV}$ and 10 $\mathrm{MeV}$ were made by instruments on Yohkoh [6], Ulysses [6], Pioneer Venus Orbiter [8], and the Compton Gamma Ray Observatory (CGRO). In this work we use data from the BATSE [1] and COMPTEL [11] instruments on CGRO.

Four of the eight BATSE detectors were exposed to the event, with $97 \%, 50 \%$, $37 \%$ and $11 \%$ of their respective areas facing the Sun. Data from these detectors are available between $\sim 25 \mathrm{keV}$ and $1.9 \mathrm{MeV}$, though we only utilize those data up to $400 \mathrm{keV}$. COMPTEL detected the event only in burst mode due to the event's

CP510, The Fifth Compton Symposium, edited by M. L. McConnell and J. M. Ryan (c) 2000 American Institute of Physics 1-56396-932-7/00/\$17.00 
location $66^{\circ}$ off COMPTEL's zenith. The spacecraft's orientation with respect to the event resulted in only $7 \mathrm{gm} / \mathrm{cm}^{2}$ obstruction of the burst detectors, a relatively small mass. These COMPTEL data cover $0.6-10 \mathrm{MeV}$.

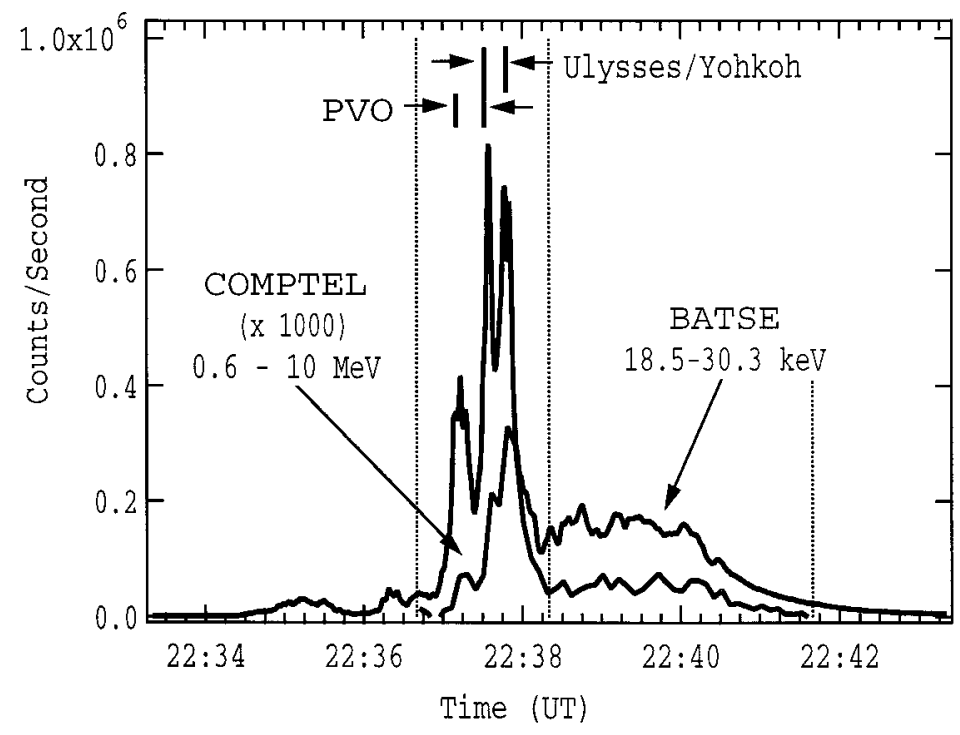

FIGURE 1. Light curve of BATSE (18.5 - 30.3 keV) and COMPTEL $(0.6-10 \mathrm{MeV})$ data (magnified by 1000.) PVO and Ulysses/Yohkoh intervals are defined between arrows.

Figure 1 shows the light curve of the flare in the two extreme energy ranges: BATSE between 18.5 and $30.3 \mathrm{keV}$ and COMPTEL integrated over the entire range of 0.6 to $10 \mathrm{MeV}$. The COMPTEL counts have been magnified by a factor of 1000 . We define two time scales: the whole event and the impulsive phase. The whole event (2236:42 - 2241:37 UT) is defined by the interval during which COMPTEL detected significant emission from the flare. The impulsive phase, which ends at 2238:24 UT, is defined as the interval of intense emission detected by COMPTEL shortly after flare onset. PVO, Ulysses and Yohkoh data used in our composite spectrum were taken during two short intervals during the impulsive phase, PVO during the first interval (2237:10 - 2237:30 UT), Yohkoh and Ulysses during the second (2237:42 - 2241:37 UT.)

\section{DATA ANALYSIS AND DISCUSSION}

\section{Composite Spectrum}

A composite spectrum of $\mathrm{X}$ - and gamma rays provides information on the electron and nuclear emission from this event. Using BATSE CONT data [12] for the 
whole event between 40 and $400 \mathrm{keV}$, we determined the electron bremsstrahlung contribution to have a spectral index of $-3.27 \pm 0.01$. This powerlaw is plotted in figure 2 along with powerlaws found from PVO, Ulysses and Yohkoh data with respective spectral indices of $-3.37 \pm 0.05[8],-3.08$ [6], and -3.82 [6]. The amplitudes of these three lines are greater than that from BATSE because the data were integrated over shorter time periods during the impulsive phase when the flux level was most intense. COMPTEL data from $400 \mathrm{keV}$ to $2.5 \mathrm{MeV}$ cover the energy range over which nuclear reactions dominate the electron brehmstrahlung. The COMPTEL spectra are decovolved using a Maximum Entropy Method [2]. The COMPTEL spectrum in figure 2 corresponds to the impulsive phase, and shows evidence for strong nuclear lines between 1 and $2.5 \mathrm{MeV}$.

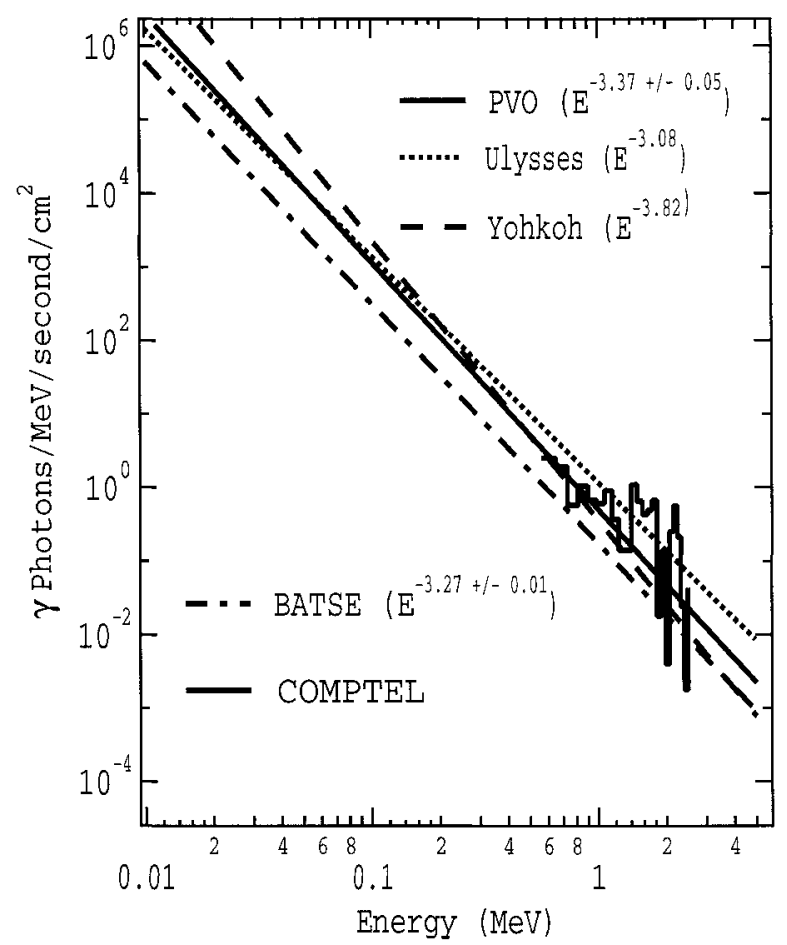

FIGURE 2. Composite spectrum with high energy data from BATSE, COMPTEL, PVO, Ulysses, and Yohkoh.

\section{Spectral Fitting}

Deconvolved spectra of the whole event and the impulsive phase are shown in figure 3 . The error bars represent $1 \sigma$ errors. The overlaid fits of the 25 data points 
were assumed to consist of a power law and either 3 or 5 gaussians. Peak locations were fixed where we expect to find common spectral lines: $0.85 \mathrm{MeV}\left({ }^{56} \mathrm{Fe}\right), 1.38$ $\mathrm{MeV}\left({ }^{24} \mathrm{Mg}\right), 1.63 \mathrm{MeV}\left({ }^{20} \mathrm{Ne}\right), 1.79 \mathrm{MeV}\left({ }^{28} \mathrm{Si}\right)$, and $2.223 \mathrm{MeV}\left({ }^{2} \mathrm{H}\right)$. The widths and amplitudes of the gaussians were free paramaters, as was the normalization factor for the power law. The $2.223 \mathrm{MeV}$ neutron capture line is well centered in both spectra. The fit of the impulsive phase spectrum converged only after removing the gaussians centered on the ${ }^{56} \mathrm{Fe}$ and ${ }^{28} \mathrm{Si}$ lines. Comparison of the two fits (recall that the impulsive phase is a subset of the whole event) suggests spectral evolution with time, as evidenced by the relative heights of the ${ }^{24} \mathrm{Mg}$ and ${ }^{20} \mathrm{Ne}$ lines in each plot.
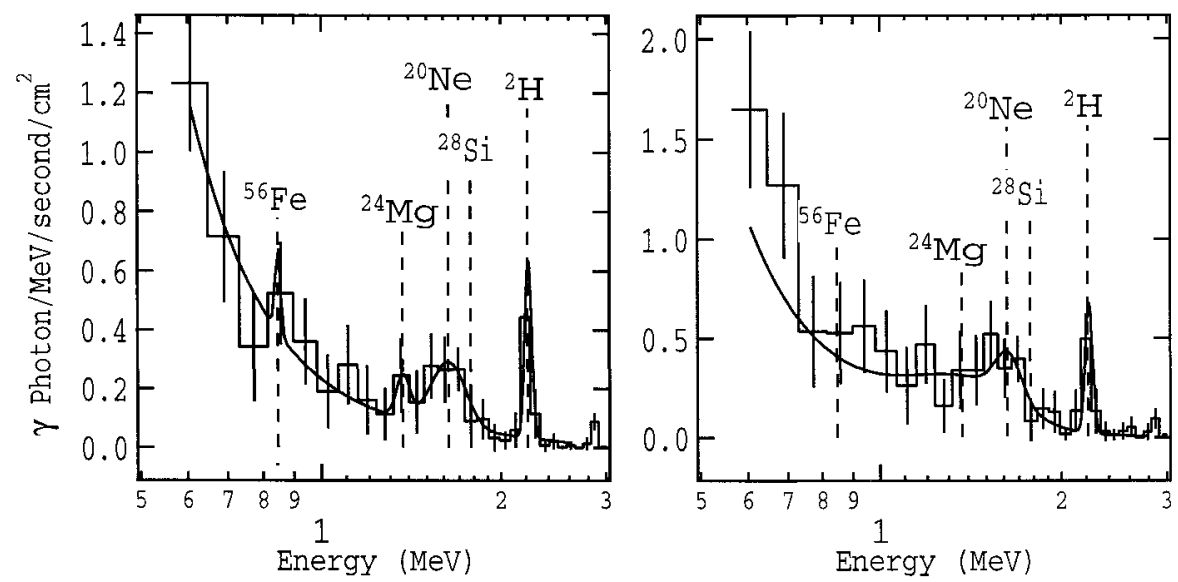

FIGURE 3. COMPTEL spectra of the whole event (left) and the impulsive phase (right). The solid lines are fits made up of a power law and either 3 or 5 guassians. Typical nuclear lines are marked.

\section{Temporal Features}

Analysis of the time evolution of the $2.223 \mathrm{MeV}$ neutron capture line after the impulsive phase shows an expected exponential decay with a best-fit time constant of $155 \pm 77$ seconds. This result is in agreement (within error bars) with time constants of $\sim 100$ seconds found by Prince et. al. [10] and Hua and Lingenfelter [3]. The time profile of the $4-7 \mathrm{MeV}$ data agrees with Yohkoh results presented by Kawabata et. al. [7] 


\section{4 - $7 \mathrm{Mev}$ to $2.223 \mathrm{MeV}$ Fluence Ratios}

COMPTEL data in the $4-7 \mathrm{MeV}$ range can be compared with the $2.223 \mathrm{MeV}$ emission to estimate the energy spectrum of the parent ions. These ions interact with heavy nuclei in the ambient solar atmosphere, resulting in gamma ray emission. For this flare, the fluence ratio is $\frac{\Phi_{4-7}}{\Phi_{2.223}}=0.45 \pm 0.11$. Combining this information with the heliocentric angle, we find a spectral index $-3.1<S<-2.6$, suggesting a hard parent ion spectrum [9] .

\section{CONCLUSIONS}

Initial results from the analysis of the high energy emission from the 1991 November 15 solar flare show a strong nuclear line component above the bremstrahlung continuum, which has a power law of $-3.27 \pm 0.01$. These nuclear lines exhibit spectral evolution with time, the 2.223 line with an expontial decay and typical time constant of $155 \pm 77$ seconds. Comparison of the $4-7$ and $2.223 \mathrm{MeV}$ line fluences suggests a hard parent ion spectrum, with a power law between -3.1 and -2.6 . The data above $2.5 \mathrm{MeV}$ need to be analyzed in more detail, in part to determine ambient solar atmosphere abundances. Data from other instruments, including interplanetary data, need to be added to this work to build a more comprehensive understanding of mechanisms at work within this flare.

\section{REFERENCES}

1. Fishman, G.J., et. al., Proceedings of the Gamma Ray Observatory Science Workshop, GSFC, MD (1989).

2. Gull, S., and Skilling, J., Quantified Maximum Entropy Users Manual, (MDEC: Meldreth) (1991).

3. Hua, X.-M., and Lingenfelter, R.E., Ap. J. 319:555-566 (1987).

4. Hudson, H. et. al., Publ. Astron. Soc. Japan 44:L77-L81 (1992).

5. Inda-Koide, M. et. al., Publ. Astron. Soc. Japan 47:661-676 (1995).

6. Kane, S.R. et. al., Ap. J. 500:1003-1008 (1998).

7. Kawabata, K. et. al., Ap. J. Supp. 90:701-705 (1994).

8. McTiernan, J. et. al., Proc of Kofu Sympsoium, NRO Report No. 360 (1994).

9. Murphy. R., Ph.D. Dissertation (1985).

10. Prince, T.A. et. al, 18th ICRC, India 4:79-82 (1983).

11. Schönfelder, V. et. al., IEEE trans. Nucl. Sci. NS-31, (1984).

12. Schwartz, R. A., personal communcation, (1999). 